\title{
Modeling and experimental investigation of a novel arc-surfaced frictional damper
}

Gongxian Wang, Yangyang Wang, Jianming Yuan, Yi Yang, Dong Wang

\begin{abstract}
In this investigation, an innovative type of frictional damper called Arc-surfaced Frictional

Damper (AFD) is introduced. The damping force of such damper is secured by pre-compression of Polyurethane Elastomer (PUE). The major advantage of AFD is that its frictional surfaces are curved so that the damping force varies with displacement.

The mathematical model for AFD is established to predict its hysteretic behavior and analyze its mechanism. Then the hysteretic behavior of AFD was studied by experimental means. Experiment program included tests with various pre-compressions at quasi-static loading and dynamic loading with various frequencies was conducted. The results show a saddle-shaped behavior in the force-displacement relation. Furthermore, it is verified that AFD does have the hysteretic property
\end{abstract}


of variable frictional force and the proposed mathematical model can be used to predict the hysteretic behavior of AFD. Hysteretic characteristics of AFD such as slip load, energy dissipation capacity, the effective stiffness and damping per cycle of loading are calculated.

Keywords: Frictional damper, mathematical model, energy dissipation, hysteretic behavior

\section{Introduction}

In the recent years, considerable studies have been investigated for structural vibration control.

Various dampers were unitized to dissipate the seismic input energy. Frictional damper is one type of such devices which dissipates the energy through frictional sliding between two solid bodies.

This kind of device reduces application costs with simple structure and enhanced reliability compared with other types of dampers $[1,2]$.

Several kinds of frictional devices have been developed in the field of protecting container cranes, buildings and bridges from seismic and wind attack. The experimental research was conducted on the slotted bolted connections (SBCs) by C.E. Grigorian [1], this device was capable of reducing structure responses. Friction joints are used by Pall and Marsh [3] to control seismic response of large panel structures. The frictional force was provided by movements between two steel bodies. Aiken and Kelly [4] suggested Sumitomo friction damper, which had the ability of self-centering. The shake table test was conducted on the multistory structure with dampers. Imad H. Mualla [5] first developed Rotational friction damper (RFD). A single story frame with RFD was studied experimentally and numerically. The damper was proved to be effective to absorb the input energy. Law [6] adopted slotted bolted connection elements to suppress seismic response of structures. The element was proved to be an effective method to control seismic response. A cylindrical 
friction damper (CFD) was proposed by Masoud Mirtaheri [7]. The frictional force was produced by movements between two steel bodies. Habib Saeed Monir [8] introduced a modified friction damper. It used rotational friction between steel stripes and bolts to provide frictional force. Lee [9] proposed a novel frictional configuration, in which load washers were used for improving energy dissipation capacity. Time history analysis results showed that the damped system was effective in reducing structural responses.

However, in most passive frictional dampers, the frictional force is pre-determined as constant value selected by designers. If a small frictional force is provided, the energy dissipated by the damper is limited under severe earthquakes. A large frictional force will lead to small drift of the damper during moderately strong and weak seismic excitations [10]. Due to the uncertainty of seismic load, the conventional friction damper cannot be controlled to adapt to this situation. Accordingly, variable friction devices have been developed to significantly control the structural vibration. Osman E. Ozbulut [11] proposed a re-centering variable friction damper (RVFD), in which a group of SMA wires was used to provide re-centering capacity. Experiment on frame structure with this damper was conducted. Hongzhe Dai [12] carried out a test on a new electromagnetic friction damper (EFD), analytical study was also conducted on the steel frame equipped with EFDs to assess its hysteretic behavior. Hamid Rahmani Samani [13] proposed a new Adjustable Frictional Damper (AFD), the frictional force was controlled by adjusting the hydraulic pressure. Liang Cao [14] proposed a Modified Friction Device (MFD) which dissipated energy via frictional mechanism caused by movements between the braking shoes and a drum. The dynamics of this device were secured using hydraulic actuation. Austin Downey [15] proposed a novel semi-active damping device called Banded Rotary Friction Device, in which the 
band can be adjusted to produce variable braking torques. The experimental results show that the damping force of this device could reach $45 \mathrm{kN}$. Its theoretical model was further validated by tests.

In spite of their efforts to develop variable friction devices suited to dissipate more seismic input energy. Their implementation is still limited. This could be due to the high maintenance costs, the unavailability of reliable technologies and complicated mechanics. Accordingly, there is still a lack of research on the frictional damper which is capable of generating large varying damping force.

The objective of this paper is to introduce an innovative arc-surfaced frictional damper (AFD) with enhanced applicability to dissipate energy. The damping force of AFD is secured by Polyurethane Elastomer (PUE). The major advantage of AFD is that it is capable of producing the force which is varying with displacement. This paper is organized as follows. First of all, the mathematical model of AFD is established to predict the damping force. The theoretical results are then validated by comparing them against the experimental data. To do this, a series of tests on AFDs were conducted. After that, a discussion including the slip force, energy dissipation capacity, the effective stiffness and equivalent viscous damping is conducted, and a comparison of the test and numerical results follows.

\section{Arc-surfaced frictional damper}

AFD mainly consists of five parts: arc plate, slider, convex plate, Polyurethane Elastomer (PUE) and T-shaped link. Fig. 1 shows a schematic illustrate of the proposed AFD. The arc plate is designed to contact with the slider, and the contact surface of both are circular. As they have the same radius, they can be well matched. Two blocks of PUE, which contact with each other, are 
shrink fitted middle two convex plates which contact with the slider through the surface of the cylinder. For this reason, the sliders and the arc plates are banded with each other with certain normal force between them, which is produced by PUE. Two sliders and two blocks of PUE are installed onto the T-shaped link which can make them move together. Also, the distance of the arc plates is getting smaller when two sliders are moving close to the end of AFD. During this process, the slider will rotate at a certain degree, which makes the slider and the plate well matched. Since the distance of the two plates is variable, the damping force will change with the moving of sliders during the motion.

Two different types of installations in the container cranes, as capable for AFD, are shown in Fig. 2. Fig. 2(a) is a diagonal brace system that transduces the deformation of the portal legs into the movement of the AFDs. Fig. 2(b) is a seesaw energy dissipation system (as configured by Kang et al. [16,17] for viscoelastic dampers and fluid viscous dampers) equipped with AFDs. The long rods are used in the mechanism, on which the pretension force is applied to keep them from compressing and buckling. When a lateral load excites the crane, the portal legs tend to sway dramatically. The beam is inclined to move horizontally, which will resist the deformation of portal legs by the combination of the structure and fictional forces. Modern container cranes weigh 1200 to $2000 \mathrm{t}$, have heights of 35 to $88 \mathrm{~m}$ above the wharf. So, this system is very suitable for the container cranes.

\section{Mathematical model of AFD}

Similar to a typical frictional damper, AFD dissipates energy by the friction between the slider and the sliding surface. However, the surface is circular and will produce variable frictional force, the radial cross-section of the surface can be defined as a function $y(x)$ in $x-y$ coordinates, as shown 
in Fig. 3.

For simplicity, the mathematical model of AFD is descripted as the slider moving along the arc plate. There are four forces acting on the slider of AFD (see Fig. 3). Considering the symmetry of the arc plate along the $x$-axis, the model consists of only one sliding surface, whose function is

$$
y(x)=\sqrt{R^{2}-x^{2}}+a_{1}-R
$$

Where $R$ is the radius of the arc plate, $a_{1}$ is the value of $y$ coordinate at zero displacement of the slider.

When the slider is in its moving state, the equation of motion for the slider in the $n$ - and

$\tau$-directions are

$$
\begin{aligned}
& \sum F_{n}=0: N-P \cos \theta-F \sin \theta=m a_{n} \\
& \sum F_{\tau}=0: F \cos \theta-P \sin \theta-T=m a_{\tau}
\end{aligned}
$$

Where $\theta$ denotes the rotation angle of the slider, $F$ denotes the axial force interacting on the slider, $N$ represents the normal contact load, $T$ signifies the tangential friction load. $P$ is the vertical force providing by the shrink of PUE. The horizontal force $F$ is caused by the external load such as earthquake excitation acting on the structure onto which AFDs are installed. $m, a_{n}$, $a_{\tau}$ are the mass, normal and tangential acceleration of the slider respectively. Note that the mass $m$ is considerably small, the inertial forces, $m a_{n}$ and $m a_{\tau}$, are actually negligible.

When the inertial forces are neglected, from Eqs. (2) and (3), the following relation is obtained

$$
\begin{aligned}
& F=\frac{T}{\cos \theta}+P \tan \theta \\
& N=\frac{P}{\cos \theta}+T \tan \theta
\end{aligned}
$$

In addition,

$$
\tan \theta=y^{\prime}(x)
$$


Substituting Eq. (6) into Eqs. (4) and (5) yields

$$
\begin{gathered}
F=T \sqrt{1+y^{\prime}(x)^{2}}+P y^{\prime}(x) \\
N=P \sqrt{1+y^{\prime}(x)^{2}}+T y^{\prime}(x)
\end{gathered}
$$

Furthermore, the friction force is expressed as

$$
T=\operatorname{sgn}(\dot{x}) \mu N
$$

Where $\mu$ denotes the friction coefficient of the sliding interface. $\operatorname{sgn}(\dot{x})$ means taking the sign of $\dot{x}$.

Considering Eq. (8) and (9) yields

$$
T=\operatorname{sgn}(\dot{x}) \mu P\left(\frac{\sqrt{1+y^{\prime}(x)^{2}}}{1-\operatorname{sgn}(\dot{x}) \mu y^{\prime}(x)}\right)
$$

After substituting Eq. (10) into Eq. (7), the axial force of the slider in its working state can be expressed as

$$
F=F_{1}+F_{2}
$$

Where

$$
\begin{gathered}
F_{1}=\operatorname{sgn}(\dot{x}) \mu P\left(\frac{1+y^{\prime}(x)^{2}}{1-\operatorname{sgn}(\dot{x}) \mu y^{\prime}(x)}\right) \\
F_{2}=P y^{\prime}(x)
\end{gathered}
$$

Assuming that the slope of the sliding surface is assumed to be far smaller than 1 , i.e. $y^{\prime}(x) \ll 1$, Eq. (12) can be modified as

$$
F_{1} \approx \operatorname{sgn}(\dot{x}) \mu P
$$

Substituting Eq. (13) and (14) into Eq. (11) yields

$$
F=\operatorname{sgn}(\dot{x}) \mu P+P y^{\prime}(x)
$$

Next, throughout this work Kelvin-Voigt model $[18,19]$ is adopted to simulate the hyper-elastic behavior of PUE, the relation between the stress and strain is expressed as 
Where $E$ and $\eta$ are the spring stiffness and dashpot viscosity respectively. The strain can be

expressed as

$$
\varepsilon=\frac{\delta}{L}
$$

Where $L$ signifies the thickness of PUE, $\delta$ is that of deformation. From Fig. 1, the deformation is expressed as

$$
\delta=a_{1}-y(x)+\Delta_{0}
$$

Where $\Delta_{0}$ is the pre-compression of PUE.

The force providing by the shrink PUE is expressed as

$$
P=A \sigma
$$

Where $A$ represents the area of PUE, which can be expressed as

$$
A=L_{1} L_{2}-\pi d^{2} / 4
$$

Where $L_{1}, L_{2}$ stand for the width and length of PUE, respectively, $d$ is the diameter of T-shaped link, which can obtain from the geometry of the arc plate, as shown in Fig. 1.

Considering Eqs. (16) (17) (18) (19) and (20), the force provides by the shrink PUE $P$ can be modified as

$$
P=\frac{L_{1} L_{2}-\pi d^{2} / 4}{L}\left[E\left(a_{1}-y(x)+\Delta_{0}\right)-\eta y^{\prime}(x)\right]
$$

Substituting Eq. (21) into Eq. (15) yields

$$
F=\frac{L_{1} L_{2}-\pi d^{2} / 4}{L}\left[E\left(a_{1}-y(x)+\Delta_{0}\right)-\eta y^{\prime}(x)\right]\left[\operatorname{sgn}(\dot{x}) \mu+y^{\prime}(x)\right]
$$

Eq. (22) is the friction force of the single slider acting on the arc plate, which is the approximate force-displacement equation for AFD in its working state. It can be used to predict the hysteretic behavior of AFD. Experimental study will be done to prove the validity of Eq. (22) in the 
following section.

\section{Experimental study}

\subsection{Test setup}

The main parts of AFD are shown in Fig. 4(a), the damper consists of three kinds of materials,

which are defined by Chinese Industrial Standards. The material of the arc plate is 45 steel and the sliders are made of brass. The inner surface of the arc plate and the surface of the slider needed no machining. PUE was machined to fit with the T-shaped link. The radius of the slider is $1000 \mathrm{~mm}$. The width and radius of the arc plate are $65 \mathrm{~mm}$ and $1000 \mathrm{~mm}$ respectively. When they are installed into the arc plate, two blocks of PUE will be compressed. The assembled AFD is shown in Fig. 4(b).

To obtain energy dissipation capacity and hysteric behavior of AFD, tests were carried out (see Fig. 5) on the testing machine with a capacity of $50 \mathrm{kN}$. Both the ends of AFD were installed in the load cells of the testing machine. The load cell at the bottom of the photo applied cyclic loading to the AFD with predetermined amplitudes. The data acquisition system was used to record the experimental data.

\subsection{Loading profile}

The tests were conducted on the basis of the two parameters of the pre-compression of PUE and loading frequency. The deformation of PUE indicates the variation of vertical load of friction, and it was changed from 3 to $7 \mathrm{~mm}$ with an increment of $2 \mathrm{~mm}$, as shown in Table 1 . Twenty cycles were applied to all the specimens at different amplitudes, as presented in Fig. 6. The loading frequencies were $0.05,0.1,0.5$ and $1 \mathrm{~Hz}$, in which the loading with $0.05 \mathrm{~Hz}$ is considered to be quasi-static loading. The AFDs will be used to protect the container crane structures from damage. 
The loading frequency was varied according to the vibrational frequencies of the structures. Most container cranes have a natural frequency with a range of $0.2-1 \mathrm{~Hz}[20]$. Thus, in the test, 0.05 and $1 \mathrm{~Hz}$ are adopted to be set as the minimum and maximum loading frequencies respectively.

\subsection{Test results and discussion}

The hysteretic behaviors for all specimens in cyclic loading are presented in Fig. 7. Where the positive displacement indicates pulling out and the negative value does pushing in. As one could expect, the hysteretic curves of the damper is saddle-shaped with stable hysteresis loops. Another observation is that there is a sharp slope on the upper and bottom hysteretic curves. This was induced by the inertia force of the sliders. In the curves, a step appears with the slope crossing $\mathrm{X}$-axis because there is a gap between the load cell and fixture.

\subsubsection{Slip force}

As can be seen in Fig. 7, the frictional force increases with the displacement. This is caused by the distance between the two arc surfaces getting smaller so that the compression of PUE increases. Then, the normal forces acting on the sliders increase, which results in the increasing frictional force.

The slip loads per cycle are estimated as the forces crossing the y-axis in the force-displacement curves. Fig. 8 presents the slip load versus cycle number for cases A to C. The slip loads of the first cycle for all cases have the largest deviation from the average values. This was induced by the imperfect contact between the sliders and the arc surfaces. Tables 2 to 4 compare the slip load per cycle with the average slip load for test cases A to $\mathrm{C}$ respectively. Ignoring the first cycle of each case, the slid load does not deviate from the averages by more than $\pm 15 \%$. On the other hand, the increments of the average slip force for cases B and C comparing with case A are $0.705 \mathrm{kN}$ and 
$0.919 \mathrm{kN}$ respectively. It can be concluded that the slip load under quasi-static stage has a nonlinear relation with the pre-compression of PUE. The maximal average frictional forces on the upper lines are presented in Table 5. The maximal ratio between the maximal average frictional force and the average slip force is 5.629 for case A. Also, the ratios seem to decrease with the increasing pre-compression.

In Fig. 9, the variation of slip load is shown according to frequency. The average values of slip load from 0.1 to $1 \mathrm{~Hz}$ represent the dynamic cases. As can be seen that the dynamic frictional force is slightly higher than that with the quasi-static loading of $0.05 \mathrm{~Hz}$. In Table 6, the maximal deviation of $15.4 \%$ between the dynamic frictional averages and quasi-static values occurred with the smallest pre-compression. Furthermore, the deviations between them seem to decrease with the increasing pre-compression.

\subsubsection{Energy Dissipation capacity}

A damping device aims to protect the structure from damage by absorbing the seismic input energy. Thus, the frictional damper must be capable of dissipating large amounts of energy.

Dissipated energy of AFD per cycle is written as

$$
E_{D i}=\int F_{i}|x| d t
$$

Where $E_{D i}$ is the dissipated energy in the $i$ th cycle, $F_{i}$ is the frictional force of the $i$ th cycle and $x$ is the displacement of the AFD.

As can be seen from Tables 2 to 4, the maximal difference from average is $14.906 \%$ which belongs to case B. it can be said that the damper has a stable energy dissipation capacity. The averages can reach $305.617,644.288$ and $903.312 \mathrm{~J}$ for cases A to C.

\subsubsection{Effective stiffness and damping}


In the analysis of structure with AFDs, the effective stiffness $\left(k_{e f f}\right)$ and the equivalent viscous damping $(\xi)$ are used to describe the properties of the damping device. $k_{\text {eff }}$ can be calculated from Eq. (24).

$$
k_{e f f}=\frac{\left|F^{+}\right|+\left|F^{-}\right|}{\left|d^{+}\right|+\left|d^{-}\right|}
$$

Where $F^{+}$and $F^{-}$are the peak forces at maximal displacements $d^{+}$and $d^{-}$, respectively.

The results of cyclic tests can also be used to evaluate the equivalent viscous damping of AFD.

The following equation can be used to calculate the equivalent viscous damping

$$
\xi=\frac{1}{4 \pi} \frac{E_{D i}}{E_{S i}}
$$

Where $E_{D i}$ and $E_{S i}$ are the total energy absorbed by the damper and the elastic strain energy per cycle respectively. Damping ratio of specimens were calculated using experimental load-displacement data, based on Eq. (25).

From the test results, the effective stiffness and the equivalent viscous damping per cycle for cases A to $\mathrm{C}$ are presented in Table 7. The effective stiffness deviates slightly from the average. It is observed that the damper is capable of dissipating large amounts of energy. The maximal equivalent viscous damping can reach 0.236 for case C. It can be said that AFD can be used to protect the structure from damage under earthquake excitation. For the limitation of test machine, it may be expected that these dampers can demonstrate large damping with larger configuration.

\section{Validation for the proposed model}

In this section the mathematical model of AFD proposed in Section. 3 has been verified by comparisons between the model results and values obtained from test. The results are presented in Fig. 10. Although there is a slight difference between them, especially at the corner of hysteretic displacement loops where excitation direction reverses, there is a good agreement between 
hysteretic model and experimental results overall. The difference is partly induced by the fact that the T-shaped link is sandwiched between the two blocks of PUE in the testing specimen. However, the influence is not taken into consideration in the theoretical model.

The applicability of the hysteretic model was also examined by the energy assumption. Table 8 presents a comparison between the model predictions and experimental results on energy dissipation of cases A to F. As can be seen from Fig. 10 and Table 8, there is a good correspondence between analytical and experimental results with a small fluctuation, the model can provides a satisfactory accuracy for practical application. In conclusion, Eq. (22) can be confidently used to estimate the force-displacement character of AFD proposed in this paper from the universal test results.

\section{Conclusion}

An innovating type of frictional damper which is called Arc-surfaced Frictional Damper (AFD) is proposed. The damping force of such damper is secured by pre-compression of PUE. The proposed damper is cheap, simple to build and high damping capacity. From the theoretical and experimental study, the following conclusions are draw:

(1) A mathematical model is developed to estimate the force-displacement character of AFD. A comparison shows that there is a well agreement between the theoretical and test results. So the mathematical model can be confidently used to predict the hysteretic behavior of AFD.

(2) The force-displacement curve of AFD is observed to be saddle-shaped. The frictional force increases with the increasing displacement. The slip force does not deviate from the average values by more than $\pm 15 \%$.

(3) The dynamic frictional force is slightly higher than that with the quasi-static loading and the 
deviation between their averages decreases with the increasing pre-compression of PUE.

(4) The ratios between the maximal average frictional forces on the upper lines and the average slip forces decrease with the increasing pre-compression and the maximal ratio can reach 5.629 .

(5) The averages of dissipated energy are $305.617,644.288$ and $903.312 \mathrm{~J}$ for test cases A to C, respectively. The maximal equivalent viscous damping can reach 0.236 with the pre-compression of $7 \mathrm{~mm}$. Therefore, AFD can be used to protect the structure from damage under earthquake excitation.

\section{Acknowledgements}

This research is supported by the National Science Foundation China (Project No. 51275369).

Their support is gratefully acknowledged.

\section{Author contributions}

Gongxian Wang and Yangyang Wang designed the experiment and wrote the first version of the manuscrift. Jianming Yuan analyzed the data. Yi Yang and Dong Wang provided valuable suggestions.

\section{References}

[1] C.E. Grigorian, TS Yang, E.P. Popov. Slotted bolted connection energy dissipaters. Earthquake Spectra 9 (3) (1993) 491-504.

[2] B. Wu, J. Zhang, M.S. Williams, J. Ou. Hysteretic behavior of improved Pall-typed frictional dampers. Engineering Structures 27 (2005) 1258-1267.

[3] A.S. Pall, C. Marsh, P. Fazio, Friction joints for seismic control of large panel structures, Journal Prestressed Concrete Institute 25 (6) (1980) 38-61. 
[4] Aiken ID, Kelly JM. Earthquake simulator testing and analytical studies of two energy-absorbing systems for multistory structures. Report no. UCB/EERC-90/03. Berkeley (CA): Earthquake Engineering Research Center. University of California, 1990.

[5] Imad H. Mualla, Borislav Belev. Performance of steel frames with a new friction damper device under earthquake excitation. Engineering Structures 24 (2002) 365-371.

[6] S.S. Law, Z.M. Wu, S.L. Chan. Analytical model of a slotted bolted connection element and its behaviour under dynamic load. Journal of Sound and Vibration 292 (2006) 777-787.

[7] Masoud Mirtaheri, Amir Peyman Zandi. Numerical and experimental study of hysteretic behavior of cylindrical friction dampers. Engineering Structures 33 (2011) 3647-3656.

[8] Habib Saeed Monir, Keyvan Zeynali. A modified friction damper for diagonal bracing of structures. Journal of Constructional Steel Research 87 (2013) 17-30.

[9] Chang-Hwan Lee, Jaeho Ryu, Jintak Oh, Chang-Hee Yoo, Young K. Ju. Friction between a new low-steel composite material and milled steel for SAFE Dampers. Eng Struct 122 (2016) 279-95.

[10] L. Gaul, S. Hurlebaus, J. Wirnitzer, H. Albrecht. Enhanced damping of lightweight structures by semi-active joints. Acta Mech 195 (2008) 249-261.

[11] Osman E. Ozbulut, Stefan Hurlebaus. Re-centering variable friction device for vibration control of structures subjected to near-field earthquakes. Mechanical Systems and Signal Processing 25 (2011) 2849-2862.

[12] Hongzhe Dai, Zhenpeng Liu, Wei Wang. Structural passive control on electromagnetic friction energy dissipation device. Thin-Walled Structures 58 (2012) 1-8.

[13] Hamid Rahmani Samani, Masoud Mirtaheri.Amir Peyman Zandi. Experimental and 
numerical study of a new adjustable frictional damper. Journal of Constructional Steel Research 112 (2015) 354-362.

[14] Liang Cao, Austin Downey, Simon Laflamme, Douglas Taylor, James Ricles. Variable friction device for structural control based on duo-servo vehicle brake: Modeling and experimental validation. Journal of Sound and Vibration 348 (2015) 41-56.

[15] Austin Downey, Liang Cao, Simon Laflamme, Douglas Taylor, James Ricles. High capacity variable friction damper based on band brake technology. Engineering Structures 113 (2016) 287-298.

[16] Jae-Do Kang and Hiroshi Tagawa. Seismic response of steel structures with seesaw systems using viscoelastic dampers. Earthquake Engng Struct. Dyn 42 (2013) 779-794.

[17] Jae-Do Kang, Hiroshi Tagawa. Seismic performance of steel structures with seesaw energy dissipation system using fluid viscous dampers. Engineering Structures 56 (2013) 431-442.

[18] Yasser E. Ibrahima, Justin Marshall, Finley A. Charney. A visco-plastic device for seismic protection of structures. Journal of Constructional Steel Research 63 (2007) 1515-1528.

[19] M. Sasso, G. Palmieri, D. Amodio. Application of fractional derivative models in linear viscoelastic problems. Mech Time-Depend Mater 15 (2011) 367-387.

[20] C. Oktay Azeloglu, Ahmet Sagirli, Ayse Edincliler. Mathematical modelling of the container cranes under seismic loading and proving by shake table. Nonlinear Dyn 73 (2013) 143-154.

\section{Figure captions}

Fig. 1. Schematic illustrate of AFD: (a) Longitudinal section and (b) Cross section.

Fig. 2. Installation schemes for AFD: (a) diagonal brace system and (b) seesaw energy dissipation 
system.

Fig. 3. Mathematical model of AFD.

Fig. 4. Prototype of the AFD: (a) main parts of the AFD and (b) Assembled AFD.

Fig. 5. Test setup.

Fig. 6. Displacement history for cyclic test.

Fig. 7. Experimental hysteretic force-displacement curve of AFD:(a) case A; (b) case B; (c) case C;

(d) case D; (e) case E; (f) case F.

Fig. 8. Slip force versus cycle number.

Fig. 9. Slip force versus frequency.

Fig. 10. Comparison between numerical and experimental force-displacement curves: (a) case A;

(b) case B; (c) case C; (d) case D; (e) case E; (f) case F.

Table 1 Specification of test cases

\begin{tabular}{|c|c|c|c|c|c|c|c|}
\hline Test case & $\Delta_{0}(\mathrm{~mm})$ & $L_{1}(\mathrm{~mm})$ & $L_{2}(\mathrm{~mm})$ & $L(\mathrm{~mm})$ & $a_{1}(\mathrm{~mm})$ & Stroke $(\mathrm{mm})$ & Frequency of loading $(\mathrm{Hz})$ \\
\hline Case A & 3 & 80 & 65 & 23 & 60 & \pm 60 & 0.05 \\
\hline Case B & 5 & 80 & 65 & 25 & 60 & \pm 60 & 0.05 \\
\hline Case C & 7 & 80 & 65 & 27 & 60 & \pm 60 & 0.05 \\
\hline Case D & 7 & 80 & 65 & 27 & 60 & \pm 40 & 0.1 \\
\hline Case E & 7 & 80 & 65 & 27 & 60 & \pm 40 & 0.5 \\
\hline Case F & 7 & 80 & 65 & 27 & 60 & \pm 40 & 1 \\
\hline
\end{tabular}

$\Delta_{0}=$ pre-compression of PUE; $L_{1}=$ width of PUE; $L_{2}=$ length of PUE; $L=$ thickness of PUE; $a_{1}=$ value of $y$ coordinate at zero displacement of the slider.

Table 2 Slip load and dissipated energy for each cycle of loading (case A)

\begin{tabular}{|c|c|c|c|c|}
\hline Cycle no. & $\begin{array}{c}\text { Slip load } \\
(\mathrm{kN})\end{array}$ & $\begin{array}{c}\text { Deviation } \\
\text { from } \\
\text { average }\end{array}$ & $\begin{array}{c}\text { Dissipated } \\
\text { energy(J) }\end{array}$ & $\begin{array}{c}\text { Deviation } \\
\text { from } \\
\text { average }\end{array}$ \\
\hline 1 & 1.14 & 47.860 & 350.792 & 14.782 \\
\hline 2 & 0.88 & 14.137 & 332.536 & 8.808 \\
\hline 3 & 0.87 & 12.840 & 324.346 & 6.128 \\
\hline 4 & 0.84 & 8.949 & 318.947 & 4.362 \\
\hline 5 & 0.81 & 5.058 & 313.385 & 2.542 \\
\hline 6 & 0.78 & 1.167 & 308.535 & 0.955 \\
\hline 7 & 0.77 & -0.130 & 304.582 & -0.339 \\
\hline 8 & 0.75 & -2.724 & 302.597 & -0.988 \\
\hline 9 & 0.74 & -4.021 & 302.102 & -1.150 \\
\hline 10 & 0.72 & -6.615 & 297.608 & -2.620 \\
\hline 11 & 0.72 & -6.615 & 299.148 & -2.117 \\
\hline
\end{tabular}




\begin{tabular}{|c|c|c|c|c|}
\hline 12 & 0.73 & -5.318 & 299.035 & -2.154 \\
\hline 13 & 0.73 & -5.318 & 297.678 & -2.598 \\
\hline 14 & 0.72 & -6.615 & 298.445 & -2.347 \\
\hline 15 & 0.72 & -6.615 & 297.383 & -2.694 \\
\hline 16 & 0.71 & -7.912 & 294.845 & -3.525 \\
\hline 17 & 0.7 & -9.209 & 294.265 & -3.714 \\
\hline 18 & 0.71 & -7.912 & 291.329 & -4.675 \\
\hline 19 & 0.69 & -10.506 & 291.631 & -4.576 \\
\hline 20 & 0.69 & -10.506 & 293.145 & -4.081 \\
\hline Average & 0.771 & & 305.617 & \\
\hline
\end{tabular}

Table 3 Slip load and dissipated energy for each cycle of loading (case B).

\begin{tabular}{|c|c|c|c|c|}
\hline Cycle no. & $\begin{array}{c}\text { Slip load } \\
(\mathrm{kN})\end{array}$ & $\begin{array}{c}\text { Deviation } \\
\text { from } \\
\text { average }\end{array}$ & $\begin{array}{c}\text { Dissipated } \\
\text { energy }(\mathrm{J})\end{array}$ & $\begin{array}{c}\text { Deviation } \\
\text { from } \\
\text { average }\end{array}$ \\
\hline 1 & 1.883 & 27.541 & 652.251 & 1.236 \\
\hline 2 & 1.395 & -5.488 & 616.162 & -4.365 \\
\hline 3 & 1.365 & -7.520 & 619.347 & -3.871 \\
\hline 4 & 1.350 & -8.537 & 620.008 & -3.769 \\
\hline 5 & 1.350 & -8.537 & 610.736 & -5.208 \\
\hline 6 & 1.358 & -8.028 & 605.764 & -5.979 \\
\hline 7 & 1.350 & -8.537 & 615.222 & -4.511 \\
\hline 8 & 1.358 & -8.028 & 620.816 & -3.643 \\
\hline 9 & 1.365 & -7.520 & 623.985 & -3.151 \\
\hline 10 & 1.388 & -5.996 & 618.338 & -4.028 \\
\hline 11 & 1.410 & -4.472 & 625.517 & -2.913 \\
\hline 12 & 1.410 & -4.472 & 626.606 & -2.744 \\
\hline 13 & 1.440 & -2.439 & 642.061 & -0.346 \\
\hline 14 & 1.485 & 0.610 & 645.458 & 0.182 \\
\hline 15 & 1.515 & 2.642 & 656.431 & 1.885 \\
\hline 16 & 1.598 & 8.232 & 673.107 & 4.473 \\
\hline 17 & 1.538 & 4.167 & 666.967 & 3.520 \\
\hline 18 & 1.598 & 8.232 & 691.343 & 7.303 \\
\hline 19 & 1.673 & 13.313 & 715.319 & 11.025 \\
\hline 20 & 1.695 & 14.837 & 740.323 & 14.906 \\
\hline Average & 1.476 & & 644.288 & \\
\hline & & & & \\
\hline
\end{tabular}

Table 4 Slip load and dissipated energy for each cycle of loading (case C).

\begin{tabular}{|c|c|c|c|c|}
\hline Cycle no. & $\begin{array}{c}\text { Slip load } \\
(\mathrm{kN})\end{array}$ & $\begin{array}{c}\text { Deviation } \\
\text { from } \\
\text { average }\end{array}$ & $\begin{array}{c}\text { Dissipated } \\
\text { energy }(\mathrm{J})\end{array}$ & $\begin{array}{c}\text { Deviation } \\
\text { from } \\
\text { average }\end{array}$ \\
\hline 1 & 3.39 & 41.574 & 960.511 & 6.332 \\
\hline 2 & 2.34 & -2.276 & 850.480 & -5.849 \\
\hline 3 & 2.25 & -6.035 & 854.972 & -5.351 \\
\hline 4 & 2.29 & -4.364 & 858.403 & -4.972 \\
\hline 5 & 2.34 & -2.276 & 868.031 & -3.906 \\
\hline 6 & 2.26 & -5.617 & 876.165 & -3.005 \\
\hline 7 & 2.37 & -1.023 & 888.018 & -1.693 \\
\hline 8 & 2.36 & -1.441 & 898.954 & -0.482 \\
\hline 9 & 2.23 & -6.870 & 908.603 & 0.586 \\
\hline 10 & 2.45 & 2.318 & 903.599 & 0.032 \\
\hline 11 & 2.34 & -2.276 & 909.821 & 0.721 \\
\hline 12 & 2.28 & -4.782 & 910.520 & 0.798 \\
\hline 13 & 2.36 & -1.441 & 913.659 & 1.145 \\
\hline 14 & 2.36 & -1.441 & 915.363 & 1.334 \\
\hline 15 & 2.41 & 0.647 & 906.485 & 0.351 \\
\hline 16 & 2.33 & -2.694 & 925.103 & 2.412 \\
\hline 17 & 2.38 & -0.606 & 927.957 & 2.728 \\
\hline 18 & 2.36 & -1.441 & 929.188 & 2.865 \\
\hline 19 & 2.38 & -0.606 & 927.150 & 2.639 \\
\hline 20 & 2.41 & 0.647 & 933.257 & 3.315 \\
\hline Average & 2.395 & & 903.312 & \\
\hline & & & & \\
\hline
\end{tabular}


Table 5 Maximal frictional force.

\begin{tabular}{|c|c|c|c|}
\hline Test case & Slip load $(\mathrm{kN})$ & $\begin{array}{c}\text { Maximal frictional force } \\
(\mathrm{kN})\end{array}$ & Ratio \\
\hline $\mathrm{A}$ & 0.771 & 4.34 & 5.629 \\
\hline $\mathrm{B}$ & 1.476 & 8.14 & 5.515 \\
\hline $\mathrm{C}$ & 2.395 & 11.07 & 4.622 \\
\hline
\end{tabular}

Table 6 Variation of slip force according to the pre-compression and loading frequencies.

\begin{tabular}{|c|c|c|c|c|c|c|}
\hline \multirow{3}{*}{$\begin{array}{l}\text { Pre-compression } \\
(\mathrm{mm})\end{array}$} & \multicolumn{4}{|c|}{ Frequency $(\mathrm{Hz})$} & \multirow{3}{*}{$\begin{array}{l}\text { Dynamic friction average } \\
\qquad(\mathrm{kN})\end{array}$} & \multirow[t]{3}{*}{ Deviration (\%) } \\
\hline & 0.05 & 0.1 & 0.5 & 1 & & \\
\hline & \multicolumn{4}{|c|}{ Slip load (kN) } & & \\
\hline 3 & 0.771 & 0.842 & 0.894 & 0.935 & 0.89 & 15.4 \\
\hline 5 & 1.476 & 1.613 & 1.68 & 1.755 & 1.68 & 13.8 \\
\hline 7 & 2.395 & 2.694 & 2.57 & 2.778 & 2.68 & 11.9 \\
\hline
\end{tabular}

Table 7 Effective stiffness and damping.

\begin{tabular}{|c|c|c|c|c|c|c|}
\hline \multirow{2}{*}{ Cycle no. } & \multicolumn{2}{|c|}{ Case A } & \multicolumn{2}{c|}{ Case B } & \multicolumn{2}{c|}{ Case C } \\
\cline { 2 - 7 } & $\begin{array}{c}\text { Effective } \\
\text { stiffness }(\mathrm{kN} / \mathrm{m})\end{array}$ & damping & $\begin{array}{c}\text { Effective } \\
\text { stiffness }(\mathrm{kN} / \mathrm{m})\end{array}$ & damping & $\begin{array}{c}\text { Effective } \\
\text { stiffness } \\
(\mathrm{kN} / \mathrm{m})\end{array}$ & damping \\
\hline 1 & 64.850 & 0.239 & 135.338 & 0.213 & 157.055 & 0.271 \\
\hline 2 & 65.124 & 0.226 & 135.402 & 0.201 & 148.167 & 0.254 \\
\hline 3 & 66.513 & 0.216 & 133.976 & 0.204 & 147.287 & 0.257 \\
\hline 4 & 66.852 & 0.211 & 136.313 & 0.201 & 158.324 & 0.240 \\
\hline 5 & 68.165 & 0.203 & 136.325 & 0.198 & 167.619 & 0.229 \\
\hline 6 & 68.610 & 0.199 & 134.822 & 0.199 & 166.385 & 0.233 \\
\hline 7 & 68.588 & 0.196 & 135.854 & 0.200 & 168.314 & 0.233 \\
\hline 8 & 68.361 & 0.196 & 135.404 & 0.203 & 172.048 & 0.231 \\
\hline 9 & 67.879 & 0.197 & 138.473 & 0.199 & 176.705 & 0.227 \\
\hline 10 & 69.165 & 0.190 & 137.185 & 0.199 & 176.446 & 0.227 \\
\hline 11 & 72.335 & 0.183 & 139.083 & 0.199 & 168.431 & 0.239 \\
\hline 12 & 70.712 & 0.187 & 134.109 & 0.207 & 173.083 & 0.233 \\
\hline 13 & 70.697 & 0.186 & 136.235 & 0.208 & 174.955 & 0.231 \\
\hline 14 & 73.459 & 0.180 & 140.958 & 0.203 & 178.550 & 0.227 \\
\hline 15 & 72.038 & 0.183 & 141.888 & 0.205 & 167.982 & 0.239 \\
\hline 16 & 71.399 & 0.183 & 140.117 & 0.212 & 173.499 & 0.236 \\
\hline 17 & 72.255 & 0.180 & 144.002 & 0.205 & 173.278 & 0.237 \\
\hline 18 & 72.619 & 0.177 & 141.077 & 0.217 & 179.859 & 0.229 \\
\hline 19 & 72.277 & 0.178 & 142.380 & 0.222 & 191.994 & 0.214 \\
\hline 20 & 72.824 & 0.178 & 139.892 & 0.234 & 178.565 & 0.231 \\
\hline Average & 69.736 & 0.194 & 137.942 & 0.207 & 169.927 & 0.236 \\
\hline
\end{tabular}

Table 8 Comparison between the model predictions and experimental results.

\begin{tabular}{|c|c|c|c|}
\hline Test cases & $\begin{array}{c}\text { Model } \\
\text { predictions }(\mathrm{J})\end{array}$ & $\begin{array}{c}\text { Experimental results } \\
\text { (average)(J) }\end{array}$ & $\begin{array}{c}\text { Deviation from } \\
\text { experimental results }\end{array}$ \\
\hline Case A & 327.79 & 305.617 & 7.255 \\
\hline Case B & 640 & 644.288 & 0.665 \\
\hline Case C & 815.78 & 903.312 & -9.69 \\
\hline Case D & 544.88 & 535.429 & 1.765 \\
\hline Case E & 544.45 & 573.7 & -5.098 \\
\hline Case F & 543.59 & 598.1 & -9.114 \\
\hline
\end{tabular}


(a)

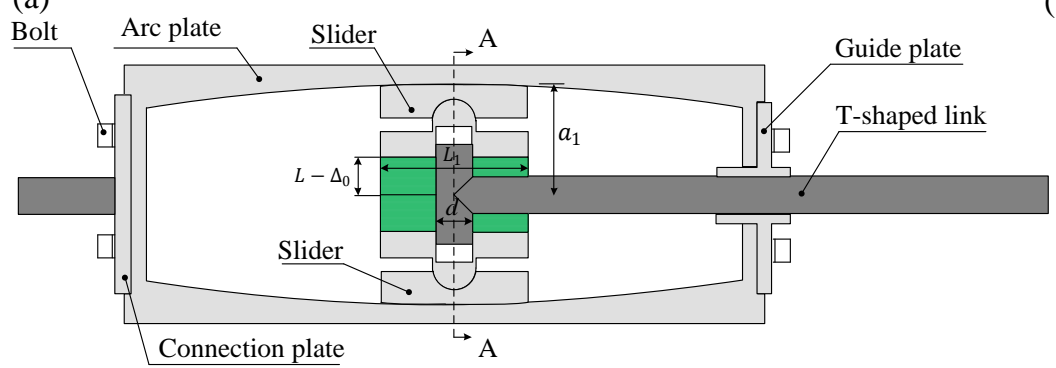

(b)

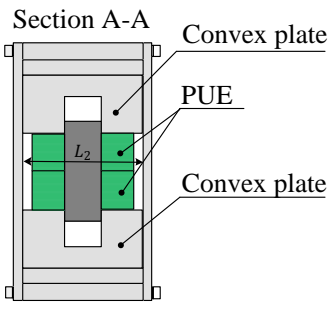

Fig. 1. Schematic illustrate of AFD: (a) Longitudinal section and (b) Cross section.

$a_{1}=$ value of $\mathrm{y}$ coordinate at zero displacement of the slider; $L=$ thickness of PUE; $\Delta_{0}=$ pre-compression of PUE; $L_{1}=$ width of PUE; $L_{2}=$ length of PUE; $d=$ diameter of T-shaped link.

(a)

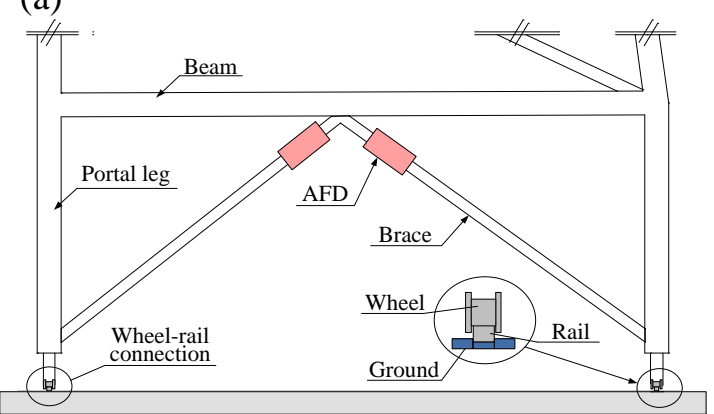

(b)

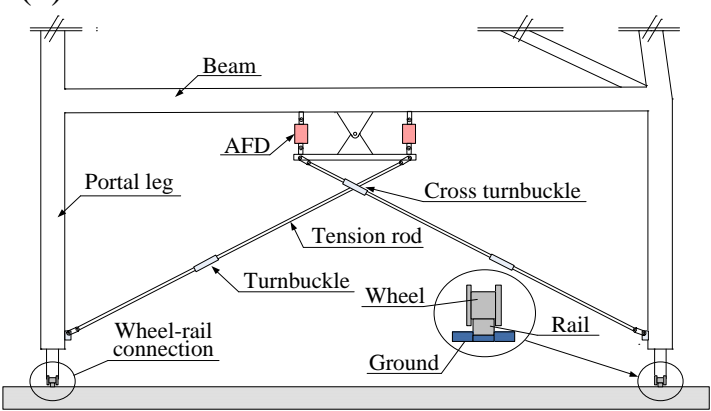

Fig. 2. Installation schemes for AFD: (a) diagonal brace system and (b) seesaw energy dissipation system.

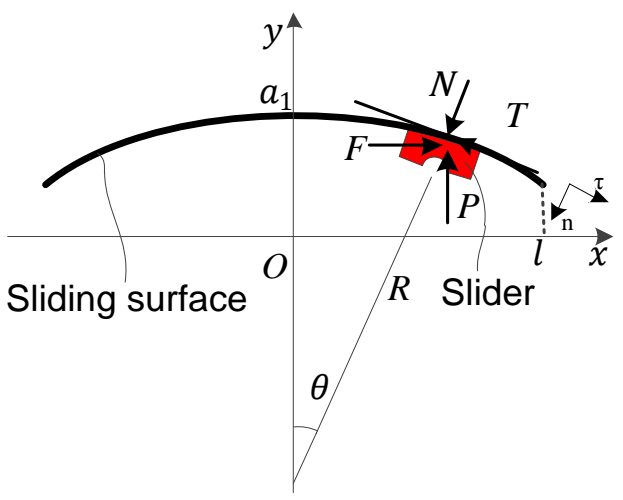

Fig. 3. Mathematical model of AFD 

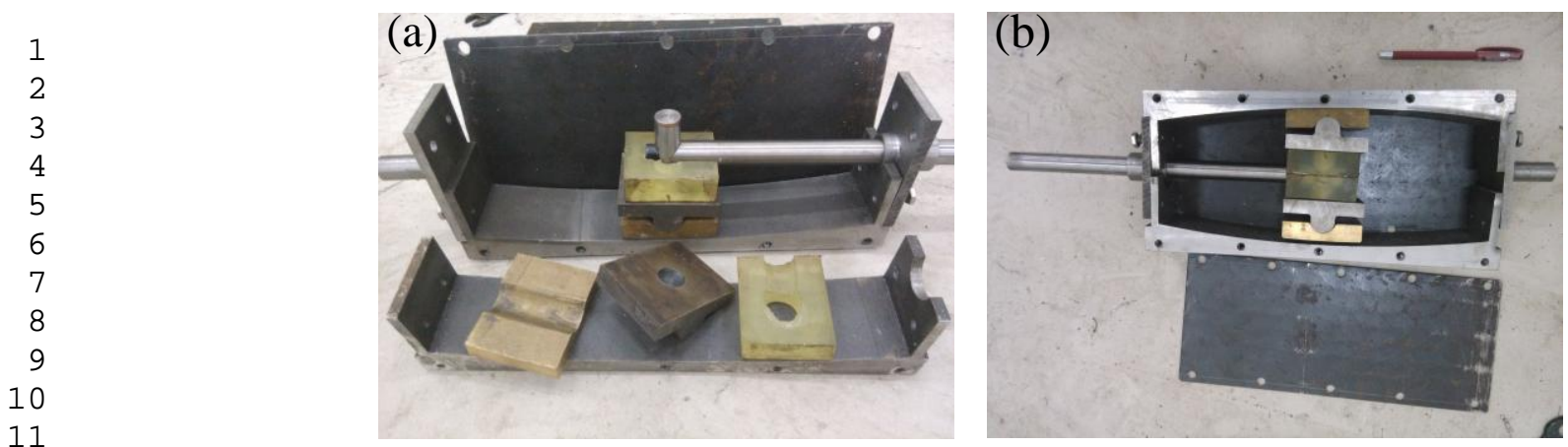

Fig. 4. Prototype of the AFD: (a) main parts of the AFD and (b) Assembled AFD.

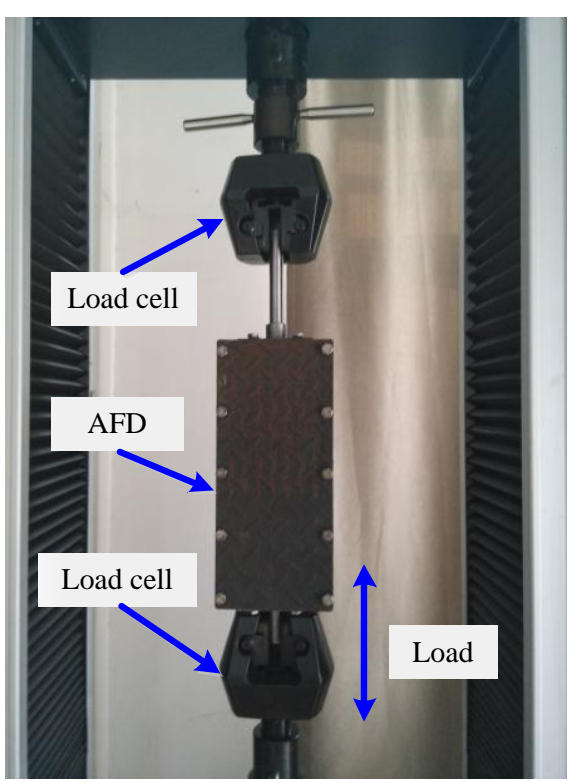

Fig. 5. Test setup

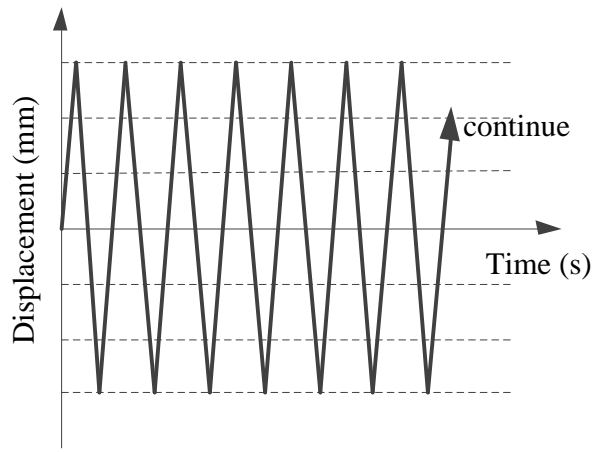

Fig. 6. Displacement history for cyclic test 

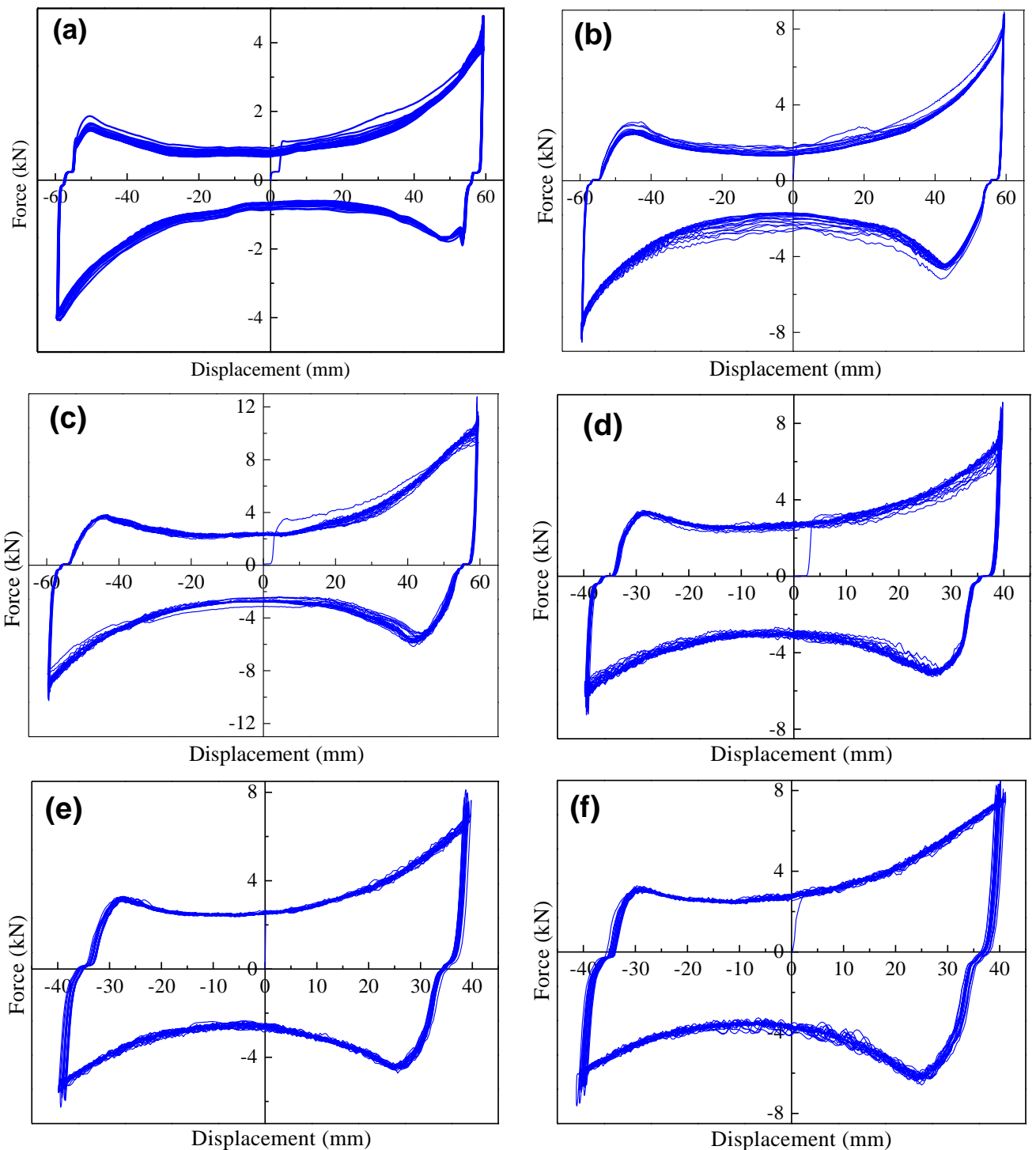

Fig. 7. Experimental hysteretic force-displacement curve of AFD: (a) case A; (b) case B; (c) case

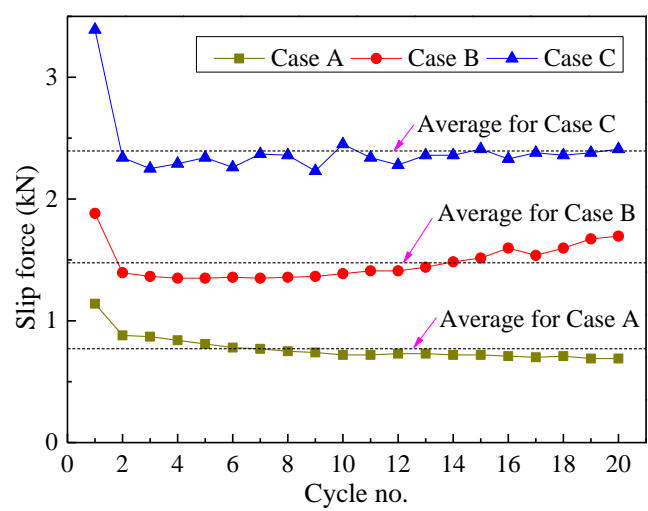

Fig. 8. Slip force versus cycle number 


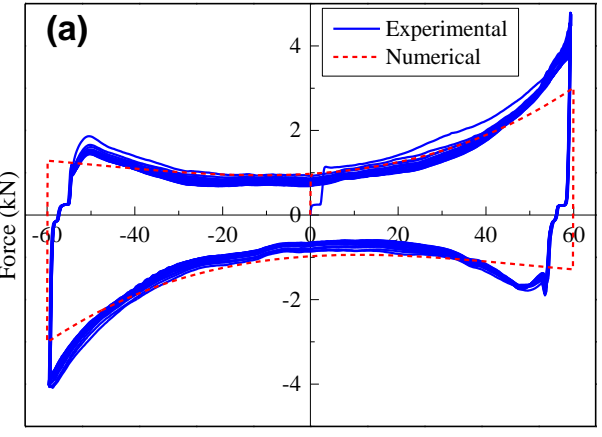

Displacement (mm)
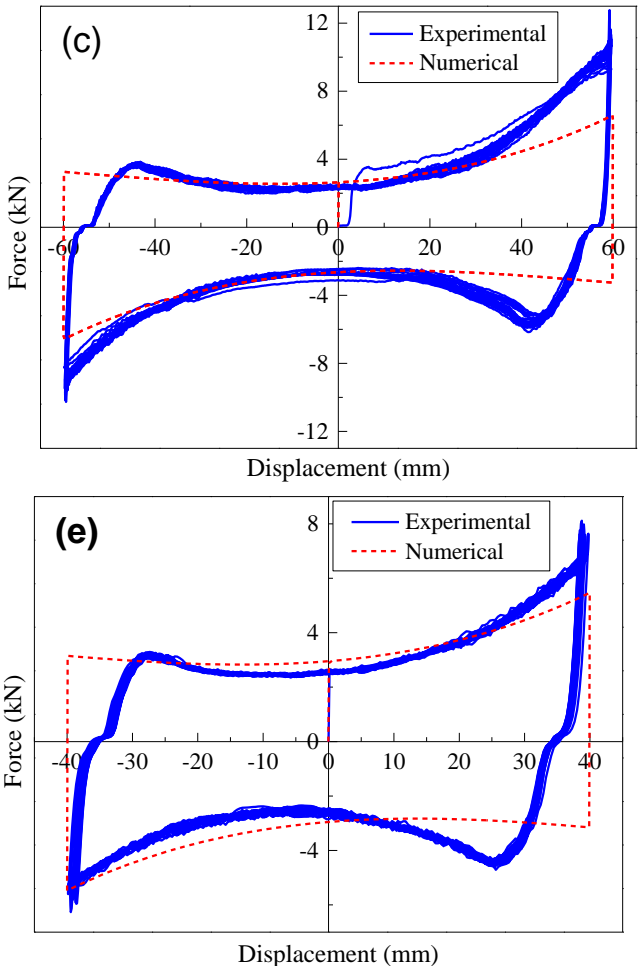

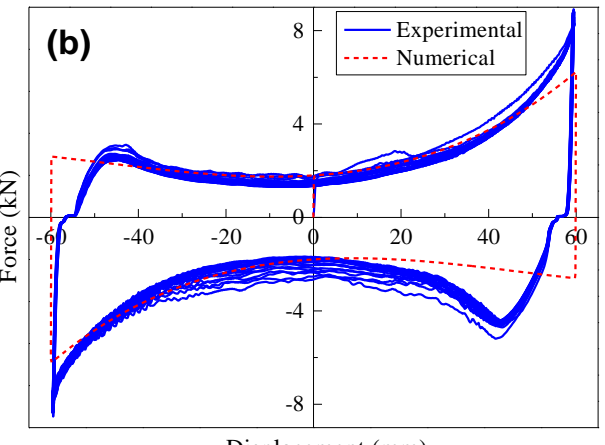

Displacement (mm)
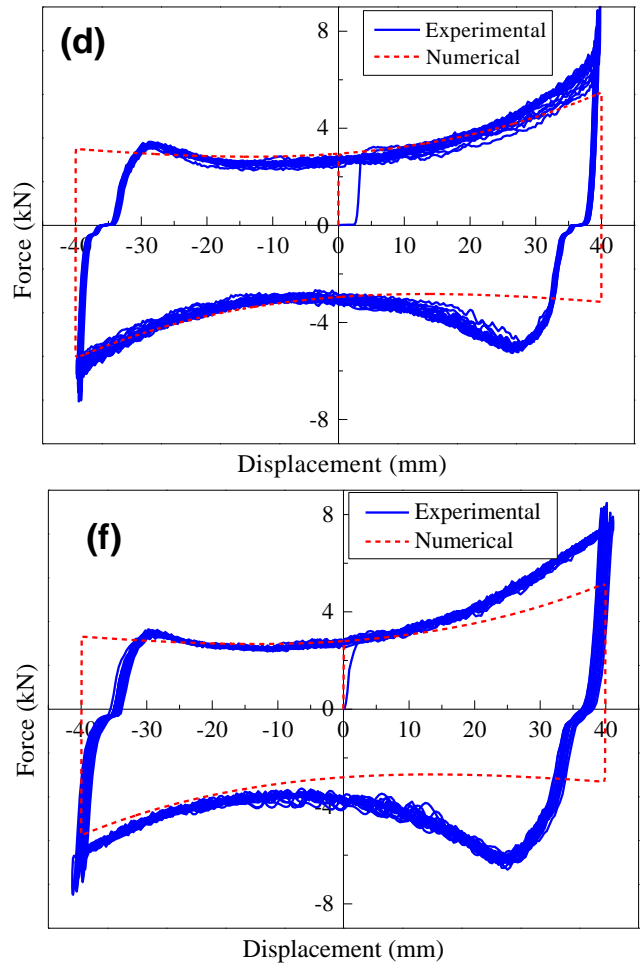

Fig. 10. Comparison between numerical and experimental force-displacement curves: (a) case A; (b) case B; (c) case C; (d) case D; (e) case E; (f) case F. 